Case Report

\title{
Mandibular Brown Tumor of Secondary Hyperparathyroidism Requiring Extensive Resection: A Forgotten Entity in the Developed World?
}

\author{
Mohammed Qaisi, ${ }^{1}$ Matthew Loeb, ${ }^{2}$ Lindsay Montague, ${ }^{3}$ and Ron Caloss ${ }^{4}$ \\ ${ }^{1}$ Oral-Head \& Neck Oncology/Microvascular Surgery, Department of Oral-Maxillofacial Surgery \& Pathology and \\ Department of Otolaryngology, Cancer Institute, University of Mississippi Medical Center, 2500 North State Street, Jackson, \\ MS 39216, USA \\ ${ }^{2}$ School of Dentistry, University of Mississippi Medical Center, 2500 North State Street, Jackson, MS 39216, USA \\ ${ }^{3}$ Oral \& Maxillofacial Pathology, Department of Oral-Maxillofacial Surgery \& Pathology, University of Mississippi Medical Center, \\ 2500 North State Street, Jackson, MS 39216, USA \\ ${ }^{4}$ Oral \& Maxillofacial Surgery, Department of Oral-Maxillofacial Surgery \& Pathology, University of Mississippi Medical Center, \\ 2500 North State Street, Jackson, MS 39216, USA
}

Correspondence should be addressed to Mohammed Qaisi; mqaisi@umc.edu

Received 7 June 2015; Accepted 5 August 2015

Academic Editor: W. Zidek

Copyright (C) 2015 Mohammed Qaisi et al. This is an open access article distributed under the Creative Commons Attribution License, which permits unrestricted use, distribution, and reproduction in any medium, provided the original work is properly cited.

\begin{abstract}
Brown tumor of hyperparathyroidism (BTHPT) is rare in the United States and not frequently seen in clinical practice. This is likely because early diagnosis and prompt treatment of this disease process prevent the progression and development of BTHPT. Conversely, BTHPT is more common in underdeveloped countries where fewer patients have access to health care and hyperparathyroidism (HPT) goes untreated. It has been reported that the incidence of BTHPT in underdeveloped countries can be as high as 58 to 69 percent in patients with primary HPT. We present a case report of a patient in the United States with a large mandibular BTHPT requiring an extensive resection in the setting of secondary HPT. Despite being rare in this country, it is important for nephrologists, primary care physicians, and oral health care providers to be able to recognize this entity, so that intervention may be rendered early.
\end{abstract}

\section{Introduction}

In a healthy individual, the parathyroid glands and kidneys play an important role in the homeostasis and regulation of serum calcium $(\mathrm{Ca})$ and phosphorus $(\mathrm{P})$ levels. Parathyroid hormone $(\mathrm{PTH})$ is released in response to decreased serum $\mathrm{Ca}$ and functions to increase this by causing an efflux of $\mathrm{Ca}$ from the bony skeleton and increased reabsorption by the kidneys. PTH also leads to increased release of vitamin $\mathrm{D}$ from the kidneys, which in turn causes increased $\mathrm{Ca}$ absorption from the gastrointestinal tract. Conversely, PTH leads to decreased P levels due to increased excretion by the kidneys [1].

Hyperparathyroidism (HPT) is a disorder characterized by an excessive amount of parathyroid hormone secretion by the parathyroid glands. Depending on the cause of this PTH production, HPT can be characterized into primary, secondary, and tertiary forms $[2,3]$.

Primary HPT occurs when one or more parathyroid glands secrete an excessive amount of PTH, as in the case of a parathyroid adenoma; secondary HPT results when increased secretion of PTH is a response to lowered ionized calcium, typically as a result of renal disease [4]. In tertiary HPT, secretion of PTH occurs as a result of long-standing chronic renal disease eventually leading to overactive parathyroid glands that become independent of the underlying disease. Hence, tertiary HPT is not corrected when patients receive a renal transplant that corrects the underlying renal etiology [5]. 


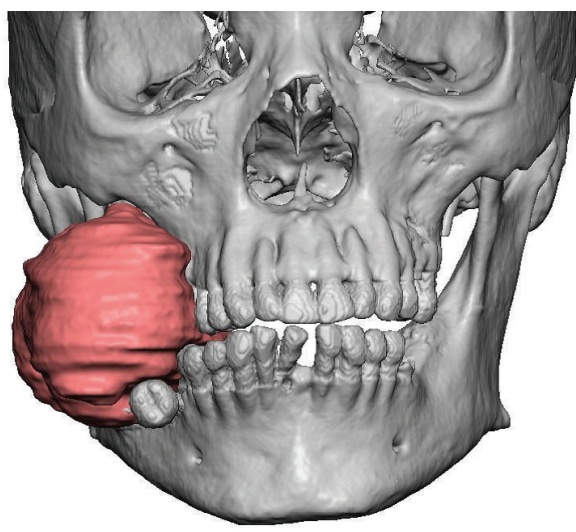

FIGURE 1: Computer simulation of patient with a large swelling on the lower right side of his face.

When any of these forms of HPT are not well controlled, BTHPT may result. These nonneoplastic lesions present late in untreated disease and are more commonly seen in underdeveloped countries due to the lack of access to care; they are infrequently seen in developed countries [6]. These lesions appear identical to central reparative giant cell granulomas histologically and result because of the abnormal calcium homeostasis in HPT [2]. Treatment for these lesions is often directed at the management of the underlying HPT, which frequently results in regression and resolution of these lesions without surgical intervention. However, surgical treatment may be required in refractory cases or in large symptomatic lesions [7].

\section{Case Report}

A 43-year-old African American male presented to the Division of Oral Oncology at the University of Mississippi Cancer Institute with the chief complaint of swelling in his right mandible that had been present for approximately one year (Figure 1). Oral exam revealed a $5.0 \times 7.0 \mathrm{~cm}$ bulbous mass arising from the mandible and obliterating the right side of the oral cavity. The mass was ulcerated due to occluding with the opposing dentition. The patient denied pain but reported difficulty in eating. A computed tomography (CT) scan (Figures 2(a) and 2(b)) showed an expansile osseous lesion, involving the entire height of the mandible and extending from the right first premolar all the way posteriorly to involve the ramus and coronoid process. This lesion caused severe bony destruction of the mandible, eroding through both buccal and lingual cortices and filling the right half of the oral cavity.

The patient's past medical history was significant for end stage renal disease (ESRD). He was on hemodialysis and was followed by a nephrologist. He had secondary HPT which was not well controlled, with a PTH level of $1,818 \mathrm{pg} / \mathrm{mL}$ at the time of presentation (normal range (NR) 11 to $77 \mathrm{pg} / \mathrm{mL}$ ). This was thought to be likely due to noncompliance with his medications and due to refractory disease. The remainder of his labs was as follows: Ca 8.4 (NR 9-10.5 mg/dL), P 5.3 (NR
3.0-4.5 mg/dL), blood urea nitroge 28 (NR 8-20 mg/dL), and creatinine 7.17 (NR 0.7-1.3 mg/dL).

Based on the past medical history, this lesion was highly suspicious for BTHPT. However, given the rarity of brown tumors and the clinical appearance and size of this lesion, other osseous and odontogenic tumors could not be excluded. An incisional biopsy was performed and histologic examination confirmed the diagnosis of BTHPT.

Microscopically, the lesion was composed of a highly cellular proliferation of bland spindle-shaped fibroblastic cells with numerous multinucleated giant cells (Figure 3 ) and spicules of woven bone. The osteoclastic giant cells were scattered throughout the cellular stroma and surrounded small spicules of bone (Figure 4). Small blood vessels, extravasated red blood cells, and focal deposits of hemosiderin were seen throughout the lesion, especially toward the periphery. The native bone showed evidence of intense bone resorption secondary to osteoclast hyperactivity and marrow fibrosis, consistent with secondary hyperparathyroidism (Figure 5). The histologic appearance of the lesion was akin to central giant cell reparative granuloma.

A discussion was held with the patient and treating nephrologist. Given the extent of the tumor and the uncontrolled underlying disease, it was decided that surgical intervention was the best option. Segmental resection with minimal margins was performed because marginal resection or enucleation procedures were deemed not feasible due to the limited amount of bone. This was done via a transcervical lip splitting approach due to limited access from the extensive size of the tumor (Figures 6-8 and supplemental video in Supplementary Material available online at http://dx.doi.org/10.1155/2015/567543). A microvascular free tissue transfer was not performed at that time because the treating nephrologist did not feel the patient could tolerate a lengthy surgery. Virtual surgical planning was utilized to help plan the mandibular resection as well as the reconstruction bar placement (Figure 9). Postoperatively, the patient's hospital course was uneventful. He was followed up by the inpatient nephrology team and continued to receive dialysis while he remained in the hospital.

The patient was followed up for several weeks after surgery, and initial healing was within normal limits. Unfortunately, the patient was noncompliant with long term followup despite very extensive efforts to get him rescheduled. This noncompliance may have potentially contributed to the initial presentation of the tumor.

\section{Discussion}

BTHPT is rare in the United States and not frequently seen in clinical practice. Conversely, BTHPT is more common in underdeveloped countries where fewer patients have access to health care and HPT goes untreated. A review of the literature over the last 10 years identified a total of 87 patients in 57 reports with BTHPT of the facial region. Analysis of this data is provided in Table 1. Only 5 of those cases occurred in the United States [8-12]. Of those, four had primary HPT, and one had secondary disease, as our patient did. The highest 


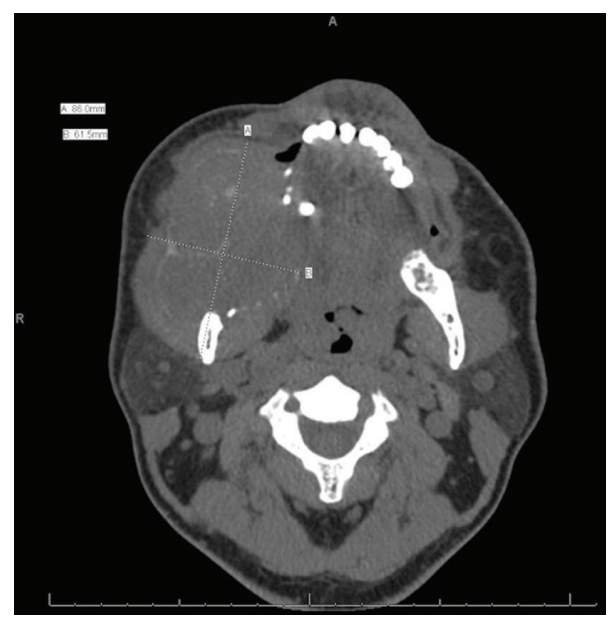

(a)

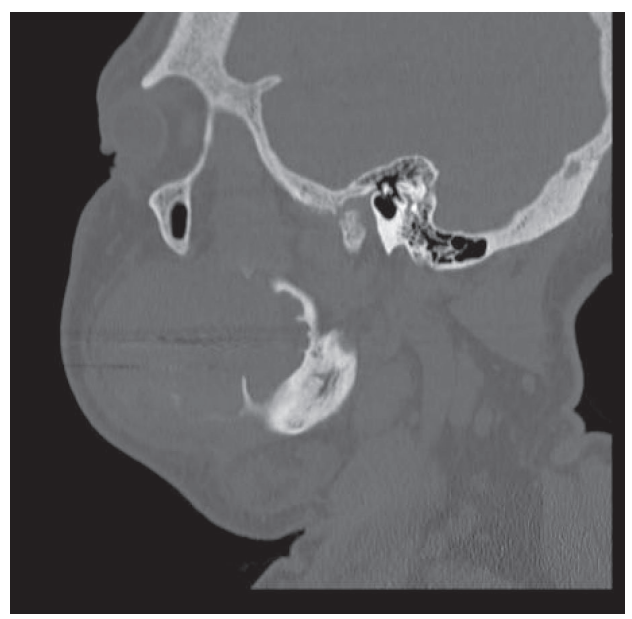

(b)

Figure 2: (a) Axial CT image. (b) CT scan, sagittal view.

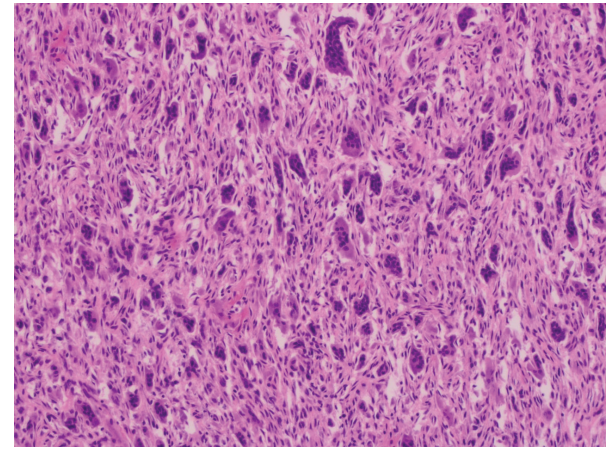

Figure 3: Photomicrograph of the mandibular brown tumor showing numerous multinucleated giant cells dispersed in a highly cellular stroma $(\mathrm{H} \& \mathrm{E}$, magnification $\times 200)$.

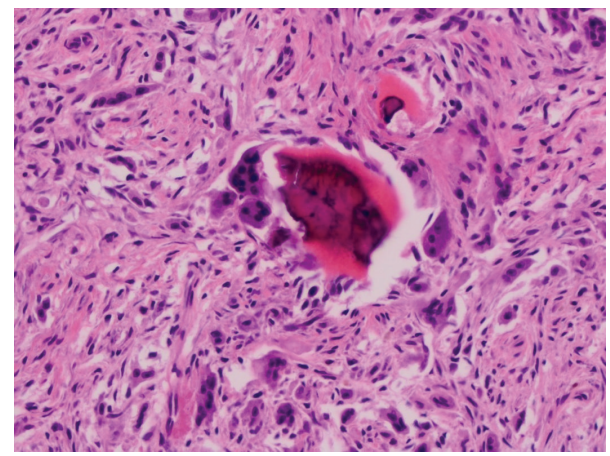

FIGURE 4: Photomicrograph showing brown tumor of hyperparathyroidism. Multinucleated giant cells surrounding a spicule of bone are visible amidst a proliferative fibroblastic stroma (H\&E, magnification $\times 400$ ).

number of BTHPTs was reported from Mexico, India, and Brazil, respectively. Of the total 87 patients identified with BTHPT, 64 were females and 23 were males $(2.8: 1)$. The mean age was 42 years (11 to 83 ). Two-thirds $(66.7 \%)$ were

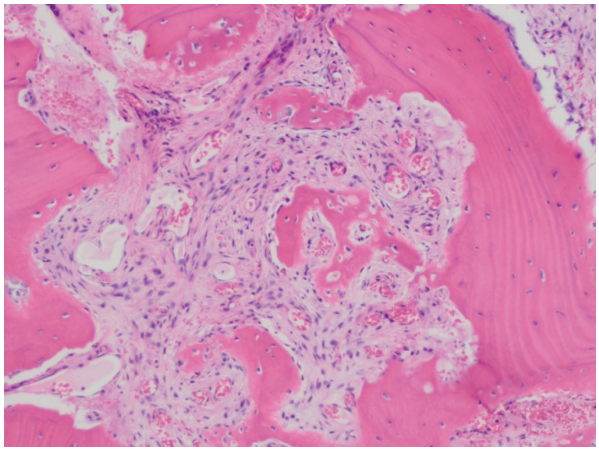

FIGURE 5: Photomicrograph showing osteoclastic bone resorption and fibrosis in secondary hyperparathyroidism (H\&E, magnification $\times 200$ ).

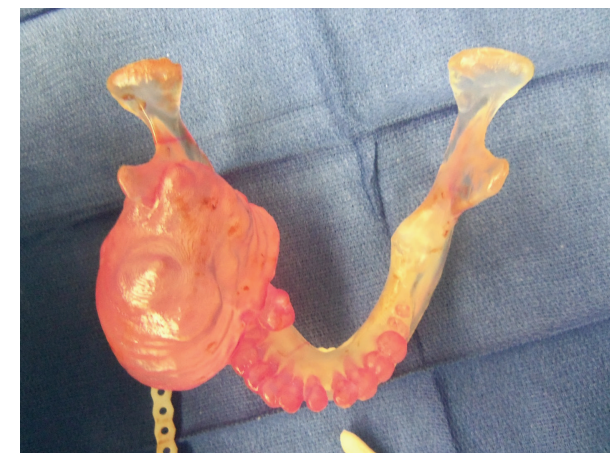

FIGURE 6: Medical model demonstrating the extent of the expansion of this lesion.

comprised of primary HPT, while secondary HPT made up $27.6 \%$ of cases; tertiary HPT made the remaining 5.7\%. With regard to site of occurrence, $48 \%$ occurred in the mandible, $38 \%$ occurred in the maxilla, and $14 \%$ involved both jaws.

BTHPT occurs late in the setting of HPT and is considered as a sign of poorly controlled disease. The prevalence of 


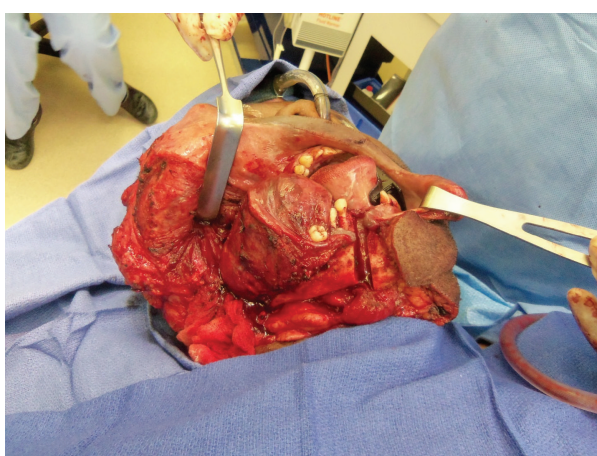

(a)

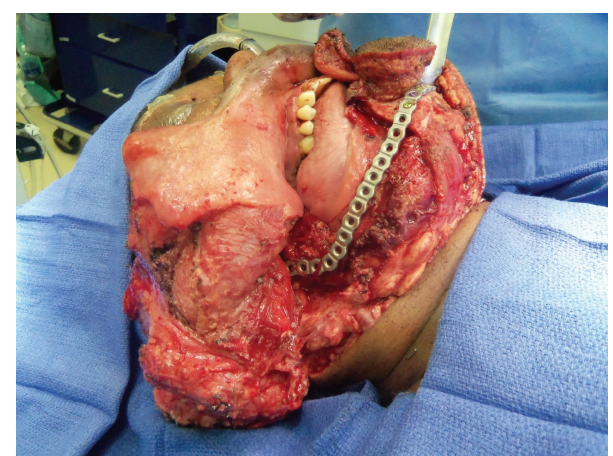

(b)

FIgURE 7: (a) Access to large brown tumor via lip split mandibulotomy approach. (b) Reconstruction bar placement.

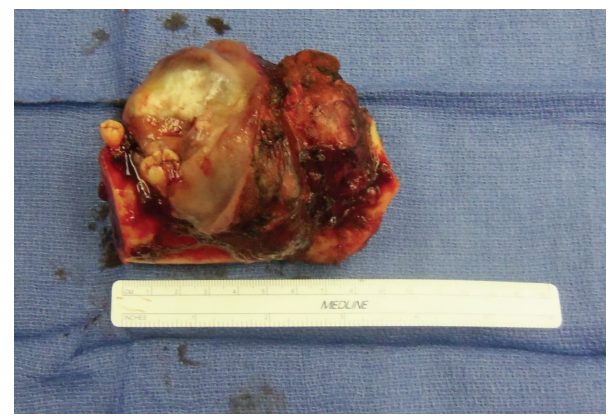

FIGURE 8: Final surgical specimen.

BTHPT is low, with a reported frequency of $3 \%$ in primary HPT and 2\% in secondary HPT [13]. Brown tumors occur due to abnormal calcium homeostasis in the setting of uncontrolled parathyroid disease, resulting in significant bone demineralization. Long-standing brown tumors, also known as osteitis fibrosa cystica, often undergo central degeneration and exhibit fibrous marrow replacement, causing a cyst-like radiographic appearance [13]. Histologically, these lesions are identical to central reparative giant cell granulomas, consisting of a spindle cell stroma and numerous multinucleated osteoclast-like giant cells. This lesion is referred to as "brown tumor" due to the reddish brown color of the tissue specimen, secondary to extravasated red blood cells and hemosiderin deposition $[7,14]$. Because it is difficult to distinguish histologically between BTHPT and other giant cell lesions, the clinical diagnosis is made based on serum chemistries and the presence or absence of HPT [15].

Primary HPT is the third most common endocrine disorder. The prevalence is reported at 1 per 1000 in the United States [5]. The most common cause of primary HPT is single gland adenoma, which represents around $90 \%$ of cases $[2,5,7,14]$. In most cases, primary HPT is diagnosed incidentally by hypercalcemia and patients are often asymptomatic [16]. However, less frequently, patients with primary HPT will display signs and symptoms of the disease. Symptoms are often related to chronic hypercalcemia and can include nephrolithiasis, muscle weakness, osteoporosis, and psychiatric symptoms $[5,14]$. Secondary HPT can be caused by either a vitamin $\mathrm{D}$ deficiency or chronic kidney disease, both of which lead to hypocalcemia and thus stimulation of the parathyroid glands. Unlike primary HPT, secondary HPT is typically associated with serum hypocalcemia and hyperphosphatemia [5, 7]. Patients with chronic kidney disease display renal osteodystrophy, which can result in bone loss, bone pain, and fractures [17].

The treatment of BTHPT varies from case to case. However, the treatment of choice related to primary HPT is most commonly to remove the offending parathyroid gland [4]. Many authors report that, after parathyroidectomy, tumor regression and healing will occur [18-22]. The most common approach to identifying the offending parathyroid gland is imaging using technetium (99mTc) scan [23]. In contrast, the treatment of secondary HPT revolves around controlling the underlying ESRD or vitamin D deficiency rather than removal of the parathyroid glands [24]. Medical management includes hemodialysis, low phosphate renal diet, treatment with calcimimetic drugs such as Cinacalcet, and vitamin $\mathrm{D}$ derivatives [1]. Renal transplantation, another option for patients with ESRD, can successfully reestablish normal kidney function [2]. Medical treatment has been shown to be sufficient in helping resolve these lesions, although the process is usually slow [25-27]. Conversely, in cases of refractory disease, parathyroidectomy may still be required, in which case total parathyroidectomy is performed with removal of all four glands. Calcium supplementation for life is required in these patients $[1,5,27]$.

Although removal of the parathyroid gland in HPT has been shown to lead to regression of the brown tumor and osseous remodeling, the patient's age can be a factor in the rate of regression $[16,20]$. When regression is slow, surgical removal of the tumor following parathyroidectomy has been shown to expedite the healing process $[24,28]$. Some have also reported tumors failing to regress and continuing to grow even after HPT has been resolved, thus requiring surgical intervention [29, 30]. Others have reported success with the use of intralesional steroid therapy to promote tumor regression; in the same manner, this method has been used for central reparative giant cell granulomas [12, 31].

Table 1 summarizes the methods of treatment for the 87 cases of maxillofacial BTHPT identified in the last 10 years, 
TABLE 1: Summary of cases of brown tumor of hyperparathyroidism in the literature.

\begin{tabular}{|c|c|c|c|c|c|c|c|c|}
\hline Author & Year & Country & Patient age & $\begin{array}{l}\text { Patient } \\
\text { gender }\end{array}$ & $\begin{array}{c}\text { Tumor } \\
\text { location }\end{array}$ & Type of HPT & Treatment & $\begin{array}{c}\text { Lesion size } \\
(\mathrm{CM})\end{array}$ \\
\hline $\begin{array}{l}\text { Chowdhury et al. } \\
{[33]}\end{array}$ & 2013 & Brazil & 20 & $\mathrm{~F}$ & $\begin{array}{l}\text { MAX \& } \\
\text { MAND }\end{array}$ & $1^{\circ} \mathrm{HPT}$ & PTE & $3 \times 3$ \\
\hline \multirow{5}{*}{ Pawlak et al. [34] } & \multirow{5}{*}{2013} & \multirow{5}{*}{ Poland } & 32 & $\mathrm{~F}$ & MAND & $1^{\circ} \mathrm{HPT}$ & $\mathrm{PTE}+\mathrm{EX} \mathrm{BT}$ & na \\
\hline & & & 57 & $\mathrm{~F}$ & MAND & $1^{\circ} \mathrm{HPT}$ & $\mathrm{PTE}+\mathrm{EX} \mathrm{BT}$ & na \\
\hline & & & 42 & $\mathrm{~F}$ & MAX & $1^{\circ} \mathrm{HPT}$ & $\mathrm{PTE}+\mathrm{EX} \mathrm{BT}$ & na \\
\hline & & & 66 & $\mathrm{~F}$ & $\begin{array}{l}\text { MAX \& } \\
\text { MAND }\end{array}$ & $1^{\circ} \mathrm{HPT}$ & PTE + EX BT & na \\
\hline & & & 32 & M & $\begin{array}{l}\text { MAX \& } \\
\text { MAND }\end{array}$ & $1^{\circ} \mathrm{HPT}$ & $\mathrm{PTE}+\mathrm{EX} \mathrm{BT}$ & na \\
\hline de Ávila et al. [35] & 2012 & Brazil & 21 & M & MAND & $3^{\circ} \mathrm{HPT}$ & PTE + CUR BT & na \\
\hline $\begin{array}{l}\text { Pace and Crosher } \\
{[3]}\end{array}$ & 2010 & UK & 27 & $\mathrm{~F}$ & $\begin{array}{l}\text { MAX \& } \\
\text { MAND }\end{array}$ & $2^{\circ} \mathrm{HPT}$ & MED MGMT & na \\
\hline Angadi et al. [36] & 2010 & India & 38 & $\mathrm{M}$ & MAND & $1^{\circ} \mathrm{HPT}$ & $\mathrm{PTE}+\mathrm{EX} \mathrm{BT}$ & $6 \times 4$ \\
\hline $\begin{array}{l}\text { Benhammou et al. } \\
\text { [37] }\end{array}$ & 2009 & France & 23 & $\mathrm{~F}$ & $\begin{array}{l}\text { MAX \& } \\
\text { MAND }\end{array}$ & $1^{\circ} \mathrm{HPT}$ & PTE & na \\
\hline Selvi et al. [38] & 2009 & Turkey & 19 & M & $\begin{array}{l}\text { MAX \& } \\
\text { MAND }\end{array}$ & $3^{\circ} \mathrm{HPT}$ & MED MGMT & $\begin{array}{c}3.45 \times 5.45 \times \\
3.46 \\
\end{array}$ \\
\hline $\begin{array}{l}\text { Karabekmez et al. } \\
\text { [39] }\end{array}$ & 2008 & Turkey & 11 & M & $\begin{array}{l}\text { MAX \& } \\
\text { MAND }\end{array}$ & $2^{\circ} \mathrm{HPT}$ & na & $15 \times 20$ \\
\hline Tarrass et al. [40] & 2008 & Morocco & 18 & $\mathrm{M}$ & MAND & $2^{\circ} \mathrm{HPT}$ & PTE & $3 \times 3$ \\
\hline Jebasingh et al. [41] & 2008 & India & 68 & $\mathrm{M}$ & MAX & $1^{\circ} \mathrm{HPT}$ & PTE & na \\
\hline Dinkar et al. [42] & 2007 & India & 36 & $\mathrm{~F}$ & MAND & $1^{\circ} \mathrm{HPT}$ & na & na \\
\hline Desigan et al. [43] & 2007 & UK & 27 & $\mathrm{~F}$ & MAND & $1^{\circ} \mathrm{HPT}$ & PTE & $2 \times 2$ \\
\hline Pinto et al. [31] & 2006 & Brazil & 12 & $\mathrm{~F}$ & MAND & $3^{\circ} \mathrm{HPT}$ & ICCT & na \\
\hline Prado et al. [27] & 2006 & Brazil & 45 & $\mathrm{~F}$ & MAND & $2^{\circ} \mathrm{HPT}$ & PTE & $2 \times 2$ \\
\hline \multirow{5}{*}{$\begin{array}{l}\text { Triantafillidou } \\
\text { et al. [7] }\end{array}$} & \multirow{5}{*}{2006} & \multirow{5}{*}{ Greece } & 76 & $\mathrm{~F}$ & $\begin{array}{l}\text { MAX \& } \\
\text { MAND }\end{array}$ & $1^{\circ} \mathrm{HPT}$ & PTE & na \\
\hline & & & 71 & M & MAND & $1^{\circ} \mathrm{HPT}$ & PTE & na \\
\hline & & & 21 & $\mathrm{~F}$ & MAND & $2^{\circ} \mathrm{HPT}$ & $\begin{array}{c}\text { CUR BT + MED } \\
\text { MGMT }\end{array}$ & na \\
\hline & & & 70 & $\mathrm{~F}$ & MAND & $2^{\circ} \mathrm{HPT}$ & $\begin{array}{c}\text { CUR BT + MED } \\
\text { MGMT }\end{array}$ & na \\
\hline & & & 68 & $\mathrm{~F}$ & MAND & $2^{\circ} \mathrm{HPT}$ & $\begin{array}{c}\text { CUR BT + MED } \\
\text { MGMT }\end{array}$ & na \\
\hline Grulois et al. [44] & 2005 & Belgium & 57 & $\mathrm{~F}$ & MAND & $1^{\circ} \mathrm{HPT}$ & na & na \\
\hline $\begin{array}{l}\text { Fernández- } \\
\text { Sanromán et al. } \\
{[45]}\end{array}$ & 2005 & Spain & 16 & $\mathrm{~F}$ & MAND & $1^{\circ} \mathrm{HPT}$ & PTE + CUR BT & na \\
\hline Jović et al. [46] & 2004 & Serbia & 25 & $\mathrm{M}$ & MAX & $2^{\circ} \mathrm{HPT}$ & $\mathrm{PTE}+\mathrm{EX} \mathrm{BT}$ & na \\
\hline $\begin{array}{l}\text { Sumer et al. } \\
{[32]}\end{array}$ & 2004 & Saudi Arabia & 41 & $\mathrm{~F}$ & MAND & $2^{\circ} \mathrm{HPT}$ & PTE & $3.5 \times 3$ \\
\hline Emin et al. [47] & 2004 & Turkey & 62 & $\mathrm{~F}$ & MAND & $1^{\circ} \mathrm{HPT}$ & $\mathrm{PTE}+\mathrm{EX}$ BT & $7 \times 5 \times 3$ \\
\hline $\begin{array}{l}\text { Suarez-Cunqueiro } \\
\text { et al. [18] }\end{array}$ & 2004 & Germany & 26 & M & MAND & $1^{\circ} \mathrm{HPT}$ & PTE & na \\
\hline Gangidi et al. [19] & 2012 & $\mathrm{UK}$ & 83 & $\mathrm{~F}$ & MAND & $1^{\circ} \mathrm{HPT}$ & PTE & na \\
\hline Placed et al. [48] & 2010 & Spain & 33 & $\mathrm{~F}$ & MAX & $2^{\circ} \mathrm{HPT}$ & na & na \\
\hline Pinto et al. [22] & 2010 & Brazil & 37 & $\mathrm{~F}$ & MAX & $2^{\circ} \mathrm{HPT}$ & PTE & na \\
\hline $\begin{array}{l}\text { Pérez-Guillermo } \\
\text { et al. [49] }\end{array}$ & 2006 & Spain & 61 & $\mathrm{M}$ & MAX & $2^{\circ} \mathrm{HPT}$ & $\mathrm{PTE}+\mathrm{EX} \mathrm{BT}$ & $3 \times 2$ \\
\hline
\end{tabular}


TABle 1: Continued.

\begin{tabular}{|c|c|c|c|c|c|c|c|c|}
\hline Author & Year & Country & Patient age & $\begin{array}{l}\text { Patient } \\
\text { gender }\end{array}$ & $\begin{array}{l}\text { Tumor } \\
\text { location }\end{array}$ & Type of HPT & Treatment & $\begin{array}{l}\text { Lesion size } \\
(\mathrm{CM})\end{array}$ \\
\hline Daniels [16] & 2004 & Saudi Arabia & 25 & $\mathrm{~F}$ & MAX & $1^{\circ} \mathrm{HPT}$ & PTE + CUR BT & na \\
\hline \multirow{22}{*}{$\begin{array}{l}\text { Reséndiz-Colosia } \\
\text { et al. [20] }\end{array}$} & \multirow{22}{*}{2008} & \multirow{22}{*}{ Mexico } & 51 & $\mathrm{~F}$ & MAX & $1^{\circ} \mathrm{HPT}$ & PTE & 5 \\
\hline & & & 27 & $\mathrm{~F}$ & MAX & $1^{\circ} \mathrm{HPT}$ & PTE & 4 \\
\hline & & & 62 & M & MAX & $1^{\circ} \mathrm{HPT}$ & PTE & 4 \\
\hline & & & 77 & $\mathrm{~F}$ & MAX & $1^{\circ} \mathrm{HPT}$ & PTE & 3 \\
\hline & & & 48 & M & MAX & $1^{\circ} \mathrm{HPT}$ & PTE & 4 \\
\hline & & & 57 & $\mathrm{~F}$ & MAX & $1^{\circ} \mathrm{HPT}$ & PTE & 4 \\
\hline & & & 64 & $\mathrm{~F}$ & MAX & $1^{\circ} \mathrm{HPT}$ & PTE & 4 \\
\hline & & & 28 & $\mathrm{~F}$ & MAND & $1^{\circ} \mathrm{HPT}$ & PTE & 3 \\
\hline & & & 55 & $\mathrm{~F}$ & MAND & $1^{\circ} \mathrm{HPT}$ & PTE & 3 \\
\hline & & & 57 & $\mathrm{~F}$ & MAND & $1^{\circ} \mathrm{HPT}$ & PTE & 4 \\
\hline & & & 41 & $\mathrm{~F}$ & MAND & $1^{\circ} \mathrm{HPT}$ & PTE & 3 \\
\hline & & & 45 & $\mathrm{~F}$ & MAND & $1^{\circ} \mathrm{HPT}$ & PTE & 2 \\
\hline & & & 68 & $\mathrm{~F}$ & MAND & $1^{\circ} \mathrm{HPT}$ & PTE & 2 \\
\hline & & & 45 & $\mathrm{~F}$ & MAND & $1^{\circ} \mathrm{HPT}$ & PTE & 5 \\
\hline & & & 41 & $\mathrm{~F}$ & MAND & $1^{\circ} \mathrm{HPT}$ & PTE & 2 \\
\hline & & & 67 & $\mathrm{~F}$ & MAND & $1^{\circ} \mathrm{HPT}$ & PTE & 2 \\
\hline & & & 53 & $\mathrm{~F}$ & MAND & $1^{\circ} \mathrm{HPT}$ & PTE & 4 \\
\hline & & & 35 & $\mathrm{~F}$ & MAND & $1^{\circ} \mathrm{HPT}$ & PTE & 2 \\
\hline & & & 42 & F & MAND & $1^{\circ} \mathrm{HPT}$ & PTE & 3 \\
\hline & & & 54 & $\mathrm{~F}$ & MAND & $1^{\circ} \mathrm{HPT}$ & PTE & 3 \\
\hline & & & 36 & $\mathrm{~F}$ & MAND & $1^{\circ} \mathrm{HPT}$ & PTE & 5 \\
\hline & & & 70 & $\mathrm{~F}$ & MAND & $1^{\circ} \mathrm{HPT}$ & PTE & 4 \\
\hline $\begin{array}{l}\text { Praveen and } \\
\text { Thriveni [50] }\end{array}$ & 2012 & India & 21 & F & $\begin{array}{l}\text { MAX \& } \\
\text { MAND }\end{array}$ & $2^{\circ} \mathrm{HPT}$ & RECON BAR & $\begin{array}{c}6 \times 7 ; 2 \times 2 \\
4 \times 2\end{array}$ \\
\hline $\begin{array}{l}\text { Arunkumar et al. } \\
\text { [51] }\end{array}$ & 2012 & India & 12 & $\mathrm{~F}$ & MAND & $2^{\circ} \mathrm{HPT}$ & $\begin{array}{c}\text { EX BT + MED } \\
\text { MGMT }\end{array}$ & na \\
\hline $\begin{array}{l}\text { Soundarya et al. } \\
{[52]}\end{array}$ & 2011 & India & 60 & M & MAX & $1^{\circ} \mathrm{HPT}$ & EX BT & na \\
\hline Jakubowski et al. [8] & 2011 & USA & 49 & $\mathrm{M}$ & MAND & $2^{\circ} \mathrm{HPT}$ & na & $8 \times 2 \times 4$ \\
\hline Sutbeyaz et al. [53] & 2009 & Turkey & 53 & M & $\begin{array}{l}\text { MAX \& } \\
\text { MAND }\end{array}$ & $1^{\circ} \mathrm{HPT}$ & PTE & $3 \times 3 ; 6 \times 7$ \\
\hline Proimos et al. [15] & 2009 & Greece & 42 & $\mathrm{~F}$ & MAX & $1^{\circ} \mathrm{HPT}$ & EX BT & 2 \\
\hline Rafizadeh et al. [10] & 2013 & USA & 43 & $\mathrm{M}$ & MAX & $1^{\circ} \mathrm{HPT}$ & na & na \\
\hline Wilson et al. [12] & 2013 & USA & 26 & $\mathrm{~F}$ & MAX & $1^{\circ} \mathrm{HPT}$ & ICT & na \\
\hline Mantar et al. [54] & 2012 & Turkey & 23 & $\mathrm{M}$ & MAX & $1^{\circ} \mathrm{HPT}$ & PTE & na \\
\hline Nabi et al. [55] & 2010 & Saudi Arabia & 24 & $\mathrm{~F}$ & MAX & $2^{\circ} \mathrm{HPT}$ & PTE & na \\
\hline Di Daniele et al. [21] & 2009 & Italy & 40 & $\mathrm{~F}$ & MAX & $2^{\circ} \mathrm{HPT}$ & PTE & 4 \\
\hline Leal et al. [24] & 2006 & Brazil & 31 & $\mathrm{~F}$ & MAX & $2^{\circ} \mathrm{HPT}$ & $\mathrm{PTE}+\mathrm{EX} \mathrm{BT}$ & na \\
\hline Oh et al. [9] & 2006 & USA & 53 & $\mathrm{~F}$ & MAX & $1^{\circ} \mathrm{HPT}$ & $\mathrm{PTE}+\mathrm{EX} \mathrm{BT}$ & 0.8 \\
\hline \multirow{2}{*}{$\begin{array}{l}\text { Pechalova and } \\
\text { Poriazova [17] }\end{array}$} & \multirow{2}{*}{2013} & \multirow{2}{*}{ Bulgaria } & 19 & $\mathrm{M}$ & MAND & $2^{\circ} \mathrm{HPT}$ & EX BT & 5 \\
\hline & & & 49 & $\mathrm{~F}$ & MAX & $2^{\circ} \mathrm{HPT}$ & EX BT & na \\
\hline Sia et al. [56] & 2012 & China & 29 & $\mathrm{~F}$ & MAX & $1^{\circ} \mathrm{HPT}$ & $\mathrm{PTE}+\mathrm{EX} \mathrm{BT}$ & $3.7 \times 4.3 \times 4.3$ \\
\hline Bahrami et al. [57] & 2012 & Iran & 38 & $\mathrm{~F}$ & MAX & $1^{\circ} \mathrm{HPT}$ & $\mathrm{PTE}+\mathrm{EX} \mathrm{BT}$ & na \\
\hline Walsh et al. [11] & 2005 & USA & 13 & $\mathrm{~F}$ & $\begin{array}{l}\text { MAX \& } \\
\text { MAND }\end{array}$ & $1^{\circ} \mathrm{HPT}$ & PTE + EX BT & na \\
\hline Di Fede et al. [58] & 2013 & Italy & 71 & M & MAND & $1^{\circ} \mathrm{HPT}$ & na & na \\
\hline
\end{tabular}


TABLE 1: Continued.

\begin{tabular}{|c|c|c|c|c|c|c|c|c|}
\hline Author & Year & Country & Patient age & $\begin{array}{l}\text { Patient } \\
\text { gender }\end{array}$ & $\begin{array}{l}\text { Tumor } \\
\text { location }\end{array}$ & Type of HPT & Treatment & $\begin{array}{l}\text { Lesion size } \\
(\mathrm{CM})\end{array}$ \\
\hline $\begin{array}{l}\text { Alhusban and } \\
\text { Baqain [59] }\end{array}$ & 2011 & Jordan & 45 & $\mathrm{~F}$ & MAND & $1^{\circ} \mathrm{HPT}$ & PTE + EX BT & na \\
\hline $\begin{array}{l}\text { Pahlavan and } \\
\text { Severin [60] }\end{array}$ & 2006 & Germany & 21 & M & MAND & $1^{\circ} \mathrm{HPT}$ & PTE & na \\
\hline Altay et al. [61] & 2013 & Turkey & 59 & $M$ & MAX & $3^{\circ} \mathrm{HPT}$ & PTE & $3.8 \times 4.6 \times 6.8$ \\
\hline Artul et al. [62] & 2013 & Israel & 46 & $\mathrm{~F}$ & MAX & $2^{\circ} \mathrm{HPT}$ & MED MGMT & 2.2 \\
\hline Guldfred et al. [63] & 2012 & Denmark & 34 & $\mathrm{~F}$ & MAX & $1^{\circ} \mathrm{HPT}$ & EX BT & na \\
\hline Nair et al. [25] & 2011 & India & 35 & $\mathrm{~F}$ & MAND & $2^{\circ} \mathrm{HPT}$ & MED MGMT & $2.7 \times 2.5 \times 1.9$ \\
\hline Magalhães et al. [64] & 2010 & Brazil & 58 & $\mathrm{~F}$ & $\begin{array}{l}\text { MAX \& } \\
\text { MAND }\end{array}$ & $3^{\circ} \mathrm{HPT}$ & PTE & na \\
\hline Pati et al. [65] & 2014 & India & 34 & $\mathrm{M}$ & MAX & $1^{\circ} \mathrm{HPT}$ & na & $5.4 \times 5.9 \times 5.2$ \\
\hline Mori et al. [66] & 2013 & Japan & 52 & $\mathrm{~F}$ & MAX & $1^{\circ} \mathrm{HPT}$ & PTE & na \\
\hline $\begin{array}{l}\text { Benjelloun et al. } \\
\text { [67] }\end{array}$ & 2007 & Morocco & 17 & $\mathrm{~F}$ & MAX & $2^{\circ} \mathrm{HPT}$ & PTE & na \\
\hline Thomas et al. [68] & 2011 & India & 27 & F & MAND & $2^{\circ} \mathrm{HPT}$ & $\begin{array}{c}\text { EX BT + MED } \\
\text { MGMT }\end{array}$ & $4 \times 6$ \\
\hline $\begin{array}{l}\text { Guerrouani et al. } \\
{[69]}\end{array}$ & 2013 & Morocco & 41 & $\mathrm{~F}$ & MAX & $1^{\circ} \mathrm{HPT}$ & PTE & na \\
\hline
\end{tabular}

Mandible $=$ MAND, maxilla $=$ MAX, hyperparathyroidism $=$ HPT, not available $=$ na, male $=\mathrm{M}$, female $=\mathrm{F}$, parathyroidectomy $=\mathrm{PTE}$, excision of brown tumor $=\mathrm{EX} \mathrm{BT}$, curettage of brown tumor $=$ CUR BT, medical management $=$ MED MGMT, intralesional corticosteroid and calcitonin therapy $=$ ICCT, mandibulectomy and reconstruction bar placement $=$ RECON BAR, and intralesional corticosteroid therapy $=$ ICT .
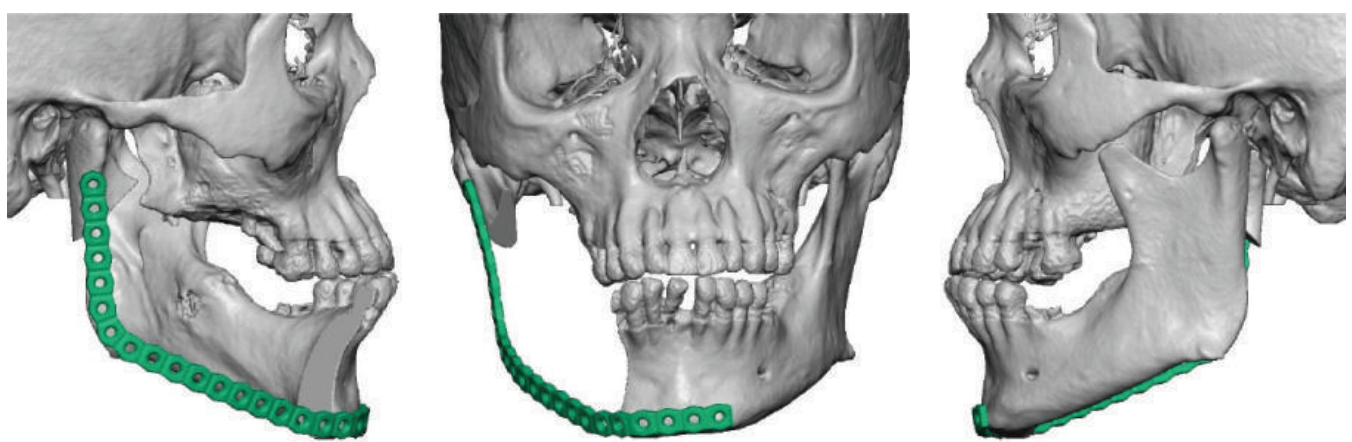

FIGURE 9: Simulated surgical procedure.

where data was available. Over $50 \%$ of patients were treated with parathyroidectomy or medical management alone. The rest had a combination of treatment modalities, although the extent of surgical curettages and excisions were difficult to assess from reviewing these reports.

In our case, surgical excision was felt to be warranted due to the refractory nature of the secondary HPT and poor compliance with medical treatment. Furthermore, the tumor was large and compromised normal function and esthetics. Patients with ESRD can have recurrence of their tumors if the renal disease is not well controlled postoperatively [32]. Our patient is at risk of developing additional brown tumors due to his poor compliance with medical therapy and the lack of surgical follow-up. We do realize that this lack of surgical follow-up is a limitation of this case report.

\section{Conclusion}

Brown tumors are rare in developed countries, with only 5 cases reported in the United States over the last 10 years at the time of this writing. These occur more frequently in developing countries because they are usually the result of uncontrolled and untreated HPT, perhaps due to lack of access to care. Regression of BTHPT can often be achieved with medical therapy or performing parathyroidectomy in primary HPT, especially if the brown tumor is small. Larger lesions, such as the one described here, usually require some sort of surgical intervention. Additionally, microvascular reconstruction may not be possible in patients with ESRD with multiple comorbidities due to the risks associated with longer anesthesia. 


\section{Conflict of Interests}

The authors declare that there is no conflict of interests regarding the publication of this paper.

\section{References}

[1] W. Saliba and B. El-Haddad, "Secondary hyperparathyroidism: pathophysiology and treatment," Journal of the American Board of Family Medicine, vol. 22, no. 5, pp. 574-581, 2009.

[2] B. W. Neville, Oral and Maxillofacial Pathology, Saunders Elsevier, St. Louis, Mo, USA, 3rd edition, 2009.

[3] C. Pace and R. Crosher, "Simultaneously occurring brown tumours in the mandible and maxilla in a patient with vitamin D deficiency," Australian Dental Journal, vol. 55, no. 4, pp. 453456, 2010 .

[4] J. S. Keyser and G. N. Postma, "Brown tumor of the mandible," American Journal of Otolaryngology-Head and Neck Medicine and Surgery, vol. 17, no. 6, pp. 407-410, 1996.

[5] W. D. Fraser, "Hyperparathyroidism," The Lancet, vol. 374, no. 9684, pp. 145-158, 2009.

[6] C. De Crea, E. Traini, L. Oragano, C. Bellantone, M. Raffaelli, and C. P. Lombardi, "Are brown tumours a forgotten disease in developed countries?" Acta Otorhinolaryngologica Italica, vol. 32, no. 6, pp. 410-415, 2012.

[7] K. Triantafillidou, L. Zouloumis, G. Karakinaris, E. Kalimeras, and F. Iordanidis, "Brown tumors of the jaws associated with primary or secondary hyperparathyroidism. A clinical study and review of the literature," The American Journal of Otolaryngology-Head and Neck Medicine and Surgery, vol. 27, no. 4, pp. 281-286, 2006.

[8] J. M. Jakubowski, I. Velez, and S. A. McClure, "Brown tumor as a result of hyperparathyroidism in an end-stage renal disease patient," Case Reports in Radiology, vol. 2011, Article ID 415476, 3 pages, 2011.

[9] A. Oh, P. Mojica, M. Sullivan et al., "Malignant hypercalcemia associated with a parathyroid macrocyst and the early genesis of a giant cell tumor," American Journal of Otolaryngology, vol. 27, no. 1, pp. 54-57, 2006.

[10] S. Rafizadeh, V. M. Duarte, C. Lai, and D. K. Chhetri, "Tumor of maxilla in a patient with hypercalcemia," JAMA Otolaryngology-Head and Neck Surgery, vol. 139, no. 7, pp. 741742, 2013 .

[11] M. D. Walsh Jr., K. Chan, S. Travers, and R. C. McIntyre Jr., "Destructive maxillomandibular brown tumor in severe hyperparathyroidism," Journal of the American College of Surgeons, vol. 201, no. 2, article 315, 2005.

[12] J. J. Wilson, H. C. Schwartz, and G. M. Tehrany, "Brown tumor of the posterior maxilla as initial manifestation of primary hyperparathyroidism: case report," Journal of Oral and Maxillofacial Surgery, vol. 71, no. 5, pp. 886-890, 2013.

[13] F. S. Chew and F. Huang-Hellinger, "Brown tumor," American Journal of Roentgenology, vol. 160, no. 4, article 752, 1993.

[14] J. A. Regezi, J. J. Sciubba, and R. C. K. Jordan, Oral Pathology: Clinical Pathologic Correlations, St. Louis, Mo, USA, Elsevier/Saunders, 6th edition, 2012.

[15] E. Proimos, T. S. Chimona, D. Tamiolakis, M. G. Tzanakakis, and C. E. Papadakis, "Brown tumor of the maxillary sinus in a patient with primary hyperparathyroidism: a case report," Journal of Medical Case Reports, vol. 3, article 7495, 2009.
[16] J. S. M. Daniels, "Primary hyperparathyroidism presenting as a palatal brown tumor," Oral Surgery, Oral Medicine, Oral Pathology, Oral Radiology and Endodontology, vol. 98, no. 4, pp. 409-413, 2004.

[17] P. F. Pechalova and E. G. Poriazova, "Brown tumor at the jaw in patients with secondary hyperparathyroidism due to chronic renal failure," Acta Medica, vol. 56, no. 2, pp. 83-86, 2013.

[18] M. M. Suarez-Cunqueiro, R. Schoen, A. Kersten, J. Klisch, and R. Schmelzeisen, "Brown tumor of the mandible as first manifestation of atypical parathyroid adenoma," Journal of Oral and Maxillofacial Surgery, vol. 62, no. 8, pp. 1024-1028, 2004.

[19] S. Gangidi, R. Dyer, and D. Cunliffe, "Not all radiolucencies of the jaw require enucleation: a case of brown tumour," British Journal of Oral and Maxillofacial Surgery, vol. 50, no. 3, pp. e33e35, 2012.

[20] J. A. Reséndiz-Colosia, S. A. Rodríguez-Cuevas, R. FloresDíaz et al., "Evolution of maxillofacial brown tumors after parathyroidectomy in primary hyperparathyroidism," Head and Neck, vol. 30, no. 11, pp. 1497-1504, 2008.

[21] N. Di Daniele, S. Condò, M. Ferrannini et al., "Brown tumour in a patient with secondary hyperparathyroidism resistant to medical therapy: case report on successful treatment after subtotal parathyroidectomy," International Journal of Endocrinology, vol. 2009, Article ID 827652, 3 pages, 2009.

[22] M. C. Pinto, S. M. G. Sass, C. P. P. Sampaio, and D. S. Campos, "Brown tumor in a patient with hyperparathyroidism secondary to chronic renal failure," Brazilian Journal of Otorhinolaryngology, vol. 76, no. 3, article 404, 2010.

[23] J. P. Bilezikian, M. L. Brandi, R. Eastell et al., "Guidelines for the management of asymptomatic primary hyperparathyroidism: summary statement from the fourth international workshop," The Journal of Clinical Endocrinology \& Metabolism, vol. 99, no. 10, pp. 3561-3569, 2014.

[24] C. T. S. Leal, P. G. S. Lacativa, E. M. S. Gomes et al., "Surgical approach and clinical outcome of a deforming brown tumor at the maxilla in a patient with secondary hyperparathyroidism due to chronic renal failure," Arquivos Brasileiros de Endocrinologia e Metabologia, vol. 50, no. 5, pp. 963-967, 2006.

[25] P. P. Nair, H. P. Gharote, S. Thomas, R. Guruprasad, and N. Singh, "Brown tumour of the jaw," BMJ Case Reports, 2011.

[26] E. Fernández, M. Belart, and J. Montoliu, "Regression of a brown tumour after 3 years of treatment with intravenous calcitriol in a haemodialysis patient," Nephrology Dialysis Transplantation, vol. 11, no. 2, pp. 398-399, 1996.

[27] F. O. Prado, A. C. D. M. N. Rosales, C. I. S. Rodrigues, R. D. Coletta, and M. A. Lopes, "Brown tumor of the mandible associated with secondary hyperparathyroidism: a case report and review of the literature," General Dentistry, vol. 54, no. 5, pp. 341-343, 2006.

[28] H. Yamazaki, Y. Ota, T. Aoki, and K. Karakida, "Brown tumor of the maxilla and mandible: progressive mandibular brown tumor after removal of parathyroid adenoma," Journal of Oral and Maxillofacial Surgery, vol. 61, no. 6, pp. 719-722, 2003.

[29] R. R. Weiss, M. J. Schoeneman, W. Primack, D. Rozycki, B. Bennett, and I. Greifer, "Maxillary brown tumor of secondary hyperparathyroidism in a hemodialysis patient," Journal of the American Medical Association, vol. 243, no. 19, pp. 1929-1930, 1980.

[30] I. Krause, B. Eisenstein, M. Davidovits, R. Cleper, A. Tobar, and S. Calderon, "Maxillomandibular brown tumor-a rare complication of chronic renal failure," Pediatric Nephrology, vol. 14, no. 6, pp. 499-501, 2000. 
[31] L. P. Pinto, K. Cherubinim, F. G. Salum, L. S. Yurgel, and M. A. Z. De Figueiredo, "Highly aggressive brown tumor in the jaw associated with tertiary hyperparathyroidism," Pediatric Dentistry, vol. 28, no. 6, pp. 543-546, 2006.

[32] A. P. Sumer, N. Arik, M. Sumer, and F. Karagoz, "A rare complication of secondary hyperparathyroidism. Brown tumor of the maxilla and mandible," Saudi Medical Journal, vol. 25, no. 12, pp. 2010-2012, 2004.

[33] S. Chowdhury, A. Aggarwal, N. Mittal, and A. Shah, "Brown tumor of hyperparathyroidism involving craniomaxillofacial region: a rare case report and literature review," Minerva Stomatologica, vol. 62, no. 9, pp. 343-348, 2013.

[34] W. Pawlak, A. Bohdanowicz-Pawlak, M. Bolanowski, J. Szymczak, G. Bednarek-Tupikowska, and K. Łuczak, "Primary hyperparathyroidism presenting as a giant cell tumor of the jaws," Neuroendocrinology Letters, vol. 34, no. 2, pp. 107-110, 2013.

[35] É. D. de Ávila, R. S. de Molon, M. A. C. Gabrielli et al., "Unusually rapid growth of brown tumour in the mandible after parathyroidectomy associated with the presence of a supernumerary parathyroid gland," Journal of Cranio-Maxillofacial Surgery, vol. 40, no. 1, pp. e19-e23, 2012.

[36] P. V. Angadi, K. Rekha, and S. R. Shetty, “An exophytic mandibular brown tumor': an unusual presentation of primary hyperparathyroidism," Oral and Maxillofacial Surgery, vol. 14, no. 1, pp. 67-69, 2010.

[37] A. Benhammou, M. Meziane, N. Dib et al., "Maxilla-mandibular brown tumors as a first sign of parathyroid adenoma," Annales d'Oto-Laryngologie et de Chirurgie Cervico-Faciale, vol. 126, no. 4, pp. 216-220, 2009.

[38] F. Selvi, S. Cakarer, R. Tanakol, S. D. Guler, and C. Keskin, "Brown tumour of the maxilla and mandible: a rare complication of tertiary hyperparathyroidism," Dentomaxillofacial Radiology, vol. 38, no. 1, pp. 53-58, 2009.

[39] F. E. Karabekmez, A. Duymaz, M. Keskin, and Z. Tosun, "Huge deforming brown tumour at the maxilla and mandible in a patient with secondary hyperparathyroidism," Journal of Plastic, Reconstructive and Aesthetic Surgery, vol. 61, no. 11, pp. 14041405, 2008.

[40] F. Tarrass, M. Benjelloun, and T. Bensaha, "Severe jaw enlargement associated with uremic hyperparathyroidism," Hemodialysis International, vol. 12, no. 3, pp. 316-318, 2008.

[41] F. Jebasingh, J. J. Jacob, A. Shah, T. V. Paul, and M. S. Seshadri, "Bilateral maxillary brown tumours as the first presentation of primary hyperparathyroidism," Oral and Maxillofacial Surgery, vol. 12, no. 2, pp. 97-100, 2008.

[42] A. D. Dinkar, S. Sahai, and M. Sharma, "Primary hyperparathyroidism presenting as an exophytic mandibular mass," Dentomaxillofacial Radiology, vol. 36, no. 6, pp. 360-363, 2007.

[43] S. Desigan, R. Syed, G. S. Conway, T. R. Kurzawinski, and J. B. Bomanji, "Giant cervical parathyroid adenoma mimicking a sternocleidomastoid mass and presenting as a brown tumor of the mandible," Clinical Nuclear Medicine, vol. 32, no. 4, pp. 306308, 2007.

[44] V. Grulois, I. Buysschaert, J. Schoenaers, F. Debruyne, P. Delaere, and V. Vander Poorten, "Brown tumour: presenting symptom of primary hyperparathyroidism," $B-E N T$, vol. 1, no. 4, pp. 191-195, 2005.

[45] J. Fernández-Sanromán, I. M. Antón-Badiola, and A. CostasLópez, "Brown tumor of the mandible as first manifestation of primary hyperparathyroidism: diagnosis and treatment," Medicina Oral, Patologia Oral y Cirugia Bucal, vol. 10, no. 2, pp. 169-172, 2005.
[46] N. Jović, R. Kozomara, S. Stosić, M. Broćić, R. Hrvacević, and S. Ilić, "Brown tumor of the maxilla in patient with secondary hyperparathyroidism," Vojnosanitetski Pregled, vol. 61, no. 6, pp. 683-687, 2004.

[47] A. H. Emin, Y. Süoğlu, D. Demir, and M. C. Karatay, "Normocalcemic hyperparathyroidism presented with mandibular brown tumor: report of a case," Auris Nasus Larynx, vol. 31, no. 3, pp. 299-304, 2004.

[48] I. G. Placed, B. Patiño-Seijas, J. Pombo-Otero, and R. AlvarezRodríguez, "Fine needle aspiration diagnosis of brown tumor of the maxilla," Acta Cytologica, vol. 54, no. 5, supplement, pp. 1076-1078, 2010.

[49] M. Pérez-Guillermo, J. Acosta-Ortega, J. García-Solano, and J. Ramos-Freixá, "Cytologic aspect of brown tumor of hyperparathyroidism. Report of a case affecting the hard palate," Diagnostic Cytopathology, vol. 34, no. 4, pp. 291-294, 2006.

[50] A. H. Praveen and R. Thriveni, "Maxillary and mandibular hyperparathyroidism," National Journal of Maxillofacial Surgery, vol. 3, no. 1, pp. 51-54, 2012.

[51] K. V. Arunkumar, S. Kumar, and D. Deepa, "Brown tumor in mandible as a first sign of vitamin D deficiency: a rare case report and review," Indian Journal of Endocrinology and Metabolism, vol. 16, no. 2, pp. 310-315, 2012.

[52] N. Soundarya, P. Sharada, N. Prakash, and G. L. Pradeep, "Bilateral maxillary brown tumors in a patient with primary hyperparathyroidism: report of a rare entity and review of literature," Journal of Oral and Maxillofacial Pathology, vol. 15, no. 1, pp. 56-59, 2011.

[53] Y. Sutbeyaz, O. Yoruk, H. Bilen, and N. Gursan, "Primary hyperparathyroidism presenting as a palatal and mandibular brown tumor," The Journal of Craniofacial Surgery, vol. 20, no. 6, pp. 2101-2104, 2009.

[54] F. Mantar, S. Gunduz, and U. R. Gunduz, "A reference finding rarely seen in primary hyperparathyroidism: brown tumor," Case Reports in Medicine, vol. 2012, Article ID 432676, 4 pages, 2012.

[55] Z. Nabi, M. Algailani, M. Abdelsalam, L. Asaad, and M. Albaqumi, "Regression of brown tumor of the maxilla in a patient with secondary hyperparathyroidism after a parathyroidectomy," Hemodialysis International, vol. 14, no. 2, pp. 247249, 2010.

[56] H.-K. Sia, M.-C. Hsieh, L.-H. Yang, and S.-T. Tu, "Maxillary brown tumor as initial presentation of parathyroid adenoma: a case report," Kaohsiung Journal of Medical Sciences, vol. 28, no. 7, pp. 400-403, 2012.

[57] E. Bahrami, T. Alireza, H. Ebrahim, and S. Mohammadreza, "Maxillary and orbital brown tumor of primary hyperparathyroidism," American Journal of Case Reports, vol. 13, pp. 183-186, 2012.

[58] V. Di Fede, O. Di Fede, V. Rodolico, G. Campisi, and A. Cordova, "Mandibular brown tumor as the first manifestation of primary hyperparathyroidism: a case report," Annali di Stomatologia, vol. 4, supplement 2, pp. 14-15, 2013.

[59] M. Alhusban and Z. H. Baqain, "Mandibular brown tumor as the first manifestation of primary hyperparathyroidism: a case report," Saudi Dental Journal, vol. 23, no. 2, pp. 107-109, 2011.

[60] P. S. Pahlavan and M. C. Severin, "Parathyroid carcinoma: a rare case with mandibular brown tumor," Wiener Klinische Wochenschrift, vol. 118, no. 5-6, pp. 175-179, 2006.

[61] C. Altay, N. Erdoğan, E. Eren, S. Altay, S. Karasu, and E. Uluç, "Computed tomography findings of an unusual maxillary 
sinus mass: brown tumor due to tertiary hyperparathyroidism," Journal of Clinical Imaging Science, vol. 3, article 55, 2013.

[62] S. Artul, A. Bowirrat, M. Yassin, and Z. Armaly, "Maxillary and frontal bone simultaneously involved in brown tumor due to secondary hyperparathyroidism in a hemodialysis patient," Case Reports in Oncological Medicine, vol. 2013, Article ID 909150, 4 pages, 2013.

[63] L.-A. Guldfred, S. Daugaard, and C. von Buchwald, "Brown tumor mimicking maxillary sinus mucocele as the first manifestation of primary hyperparathyroidism," Auris Nasus Larynx, vol. 39, no. 4, pp. 418-421, 2012.

[64] D.-P. Magalhães, R.-L. Osterne, A.-P. Alves, P.-S. S. Santos, R.B. Lima, and F.-B. Sousa, "Multiple brown tumours of tertiary hyperparathyroidism in a renal transplant recipient: a case report," Medicina Oral, Patologia Oral y Cirugia Bucal, vol. 15, no. 1, pp. e10-e13, 2010.

[65] A. R. Pati, Mubeen, K. R. Vijayalakshmi, and C. Singh, "Diagnosis and clinico-radiological presentation in an aggressive maxillary brown tumour," Journal of Clinical and Diagnostic Research, vol. 8, no. 5, pp. ZD13-ZD15, 2014.

[66] H. Mori, Y. Okada, T. Arao, S. Shimaziri, and Y. Tanaka, "A case of multiple brown tumors with primary hyperparathyroidism," Journal of Bone and Mineral Metabolism, vol. 31, no. 1, pp. 123127, 2013.

[67] M. Benjelloun, F. Tarrass, L. Alaoui et al., "Marked facial enlargement in secondary hyperparathyroidism," Nephrology Dialysis Transplantation, vol. 22, no. 10, pp. 3082-3083, 2007.

[68] S. Thomas, P. Nair, K. Hegde, and S. Neelakantan, "A bilaterally recurring exophytic mass on the lower jaw," BMJ Case Reports, vol. 2011, 2011.

[69] A. Guerrouani, A. Rzin, and K. El Khatib, "Hyperparathyroidism-jaw tumour syndrome detected by aggressive generalized osteitis fibrosa cystica," Clinical Cases in Mineral and Bone Metabolism, vol. 10, no. 1, pp. 65-67, 2013. 


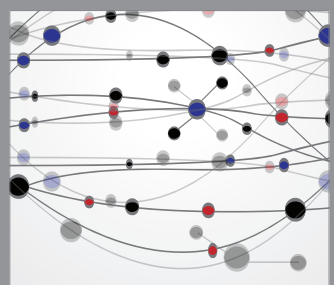

The Scientific World Journal
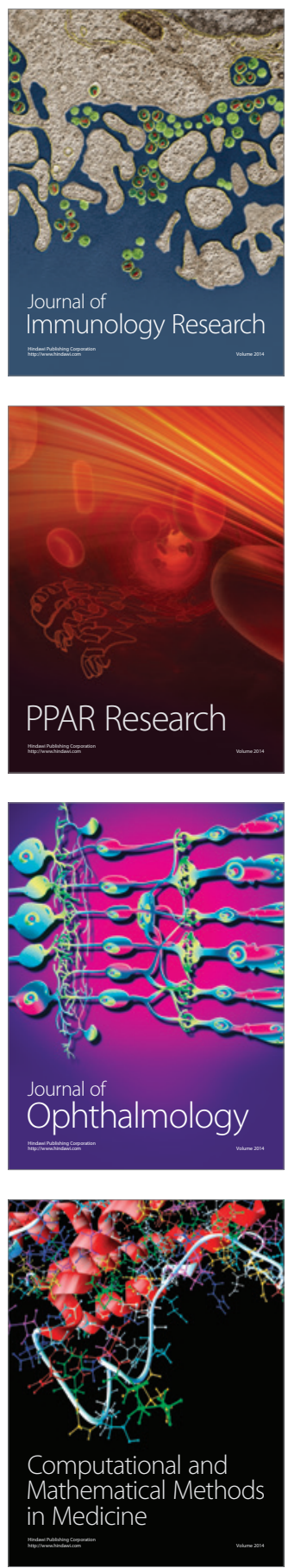

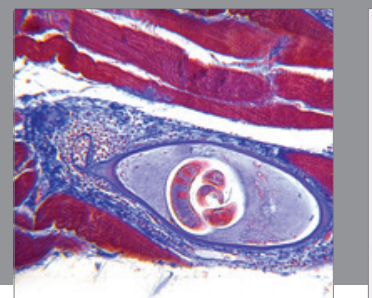

Gastroenterology

Research and Practice
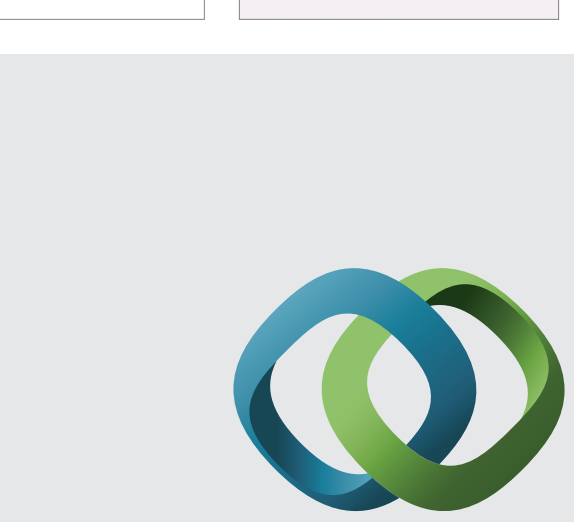

\section{Hindawi}

Submit your manuscripts at

http://www.hindawi.com
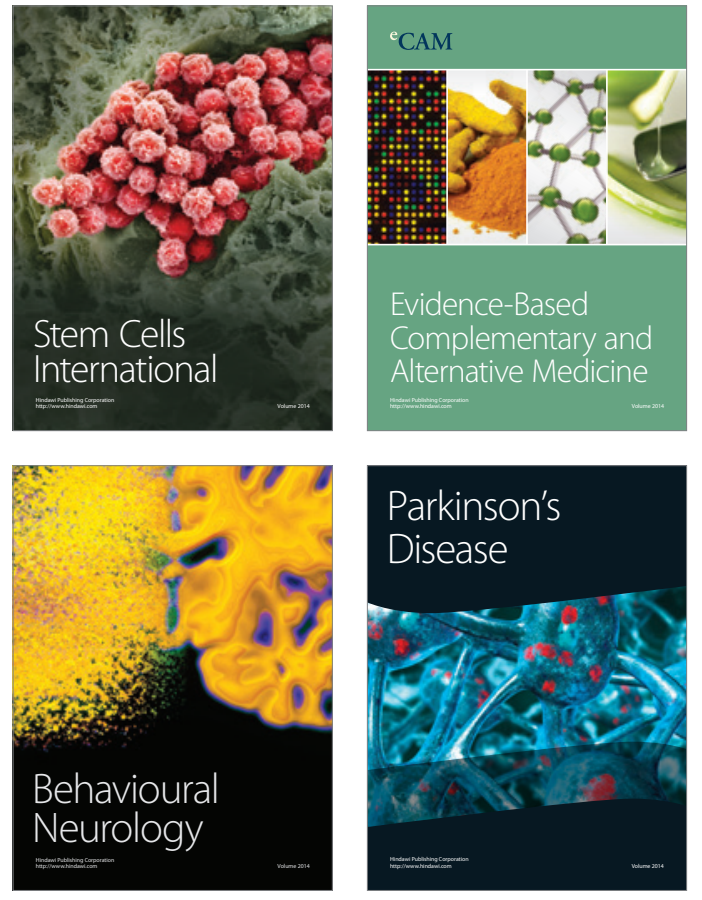
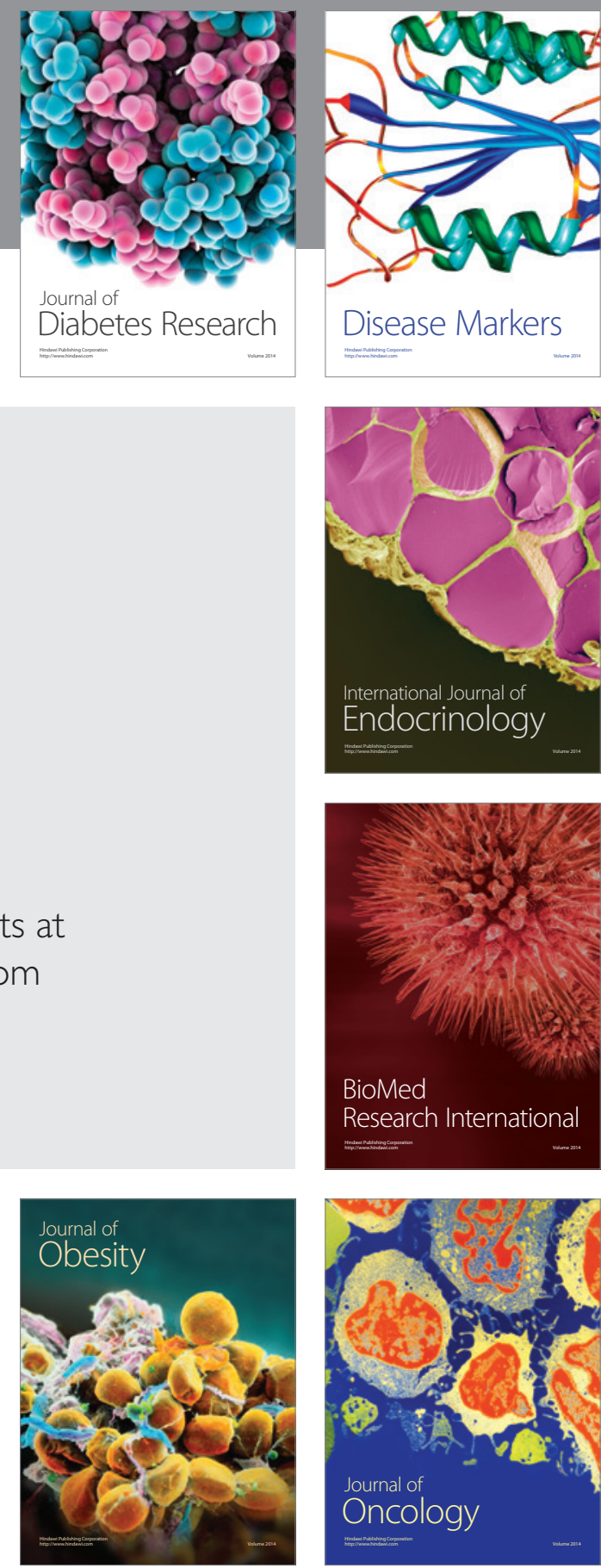

Disease Markers
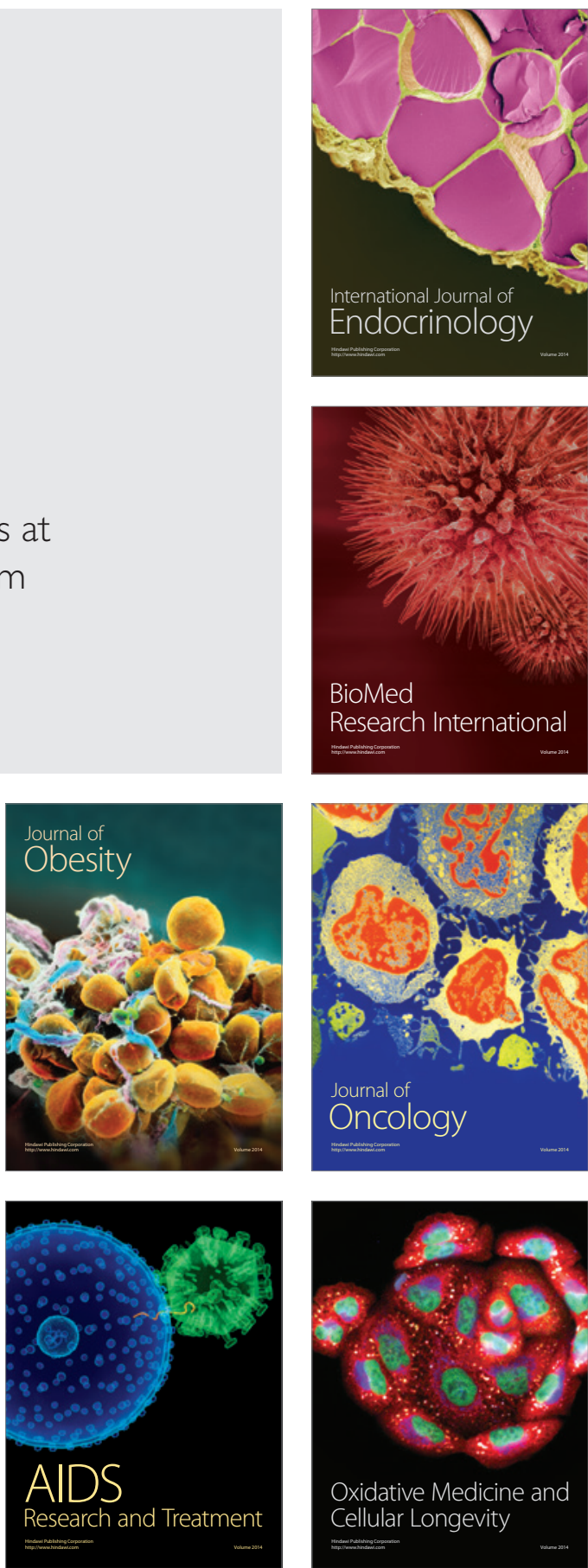